\title{
Heterosis for Yield, Yield Attributes and Water use Efficiency related Traits in Mungbean
}

\author{
B.R.K. Reddy ${ }^{*}$, K.H.P. Reddy ${ }^{2}$, D.M. Reddy ${ }^{2}$, P. Sudhakar ${ }^{2}$ and B. Ravindra Reddy ${ }^{2}$ \\ ${ }^{1}$ Regional Agricultural Research Station (RARS), Tirupati-517502, Andhra Pradesh, India \\ ${ }^{2} S$. V. Agricultural College, Tirupati, India
}

*Corresponding author

\section{A B S T R A C T}

\section{Keywords}

Mungbean,

Heterosis, Water use efficiency

Article Info

Accepted:

07 November 2019

Available Online:

10 December 2019
Twenty one $F_{1}$ crosses generated by crossing seven diverse mungbean genotypes viz., ML-267, LGG-528, MGG-390, WGG-42, AKM-9904, LM95 and EC-362096 in a $7 \times 7$ diallel fashion without reciprocals were evaluated for fourteen quantitative characters Heterosis studies conducted on relative heterosis, heterobeltiosis and standard heterosis revealed that the cross combinations viz., LM $95 \times$ EC 362096, MGG $390 \times$ LM 95 and ML $267 \times$ LGG 528 were adjudged as the best for majority of the yield, water use efficiency and heat stress tolerant traits. Hence, these crosses could be suggested for exploiting in future breeding programmes for obtaining transgressive segregants with high yield coupled with high WUE and heat tolerance.

\section{Introduction}

Mungbean popularly known as green gram [Vigna radiata (L.) Wilczek] occupies predominant position in the pulse basket in terms of dietary elements and agronomic aspects. It is a short duration grain legume having wider adaptability and is extensively grown from humid to arid and semi-arid regions. Green gram is an excellent source of high-quality protein with easy digestibility, consumed as whole grains, dal and sprouted in variety of ways. Besides being a rich source of protein, it maintains soil fertility through biological nitrogen fixation in soil and thus plays vital role in sustainable agriculture. Genetic enhancement for yield and quality seed would be a critical factor in productivity.

Among the constraints for low yield of mungbean, drought stress and heat stress are prominent. Water deficits and high temperature occur together in many environments and both stresses can interact to reduce yields. Improving the tolerance of crops to drought requires a broader 
interdisciplinary approach involving understanding factors determining yield and identification of reliable physiological traits. Thus, combination of different water use efficiency and high temperature tolerance traits are relevant, rather than a single trait used as selection criteria for screening appropriate plant ideotype. Hence, genetic enhancement for drought and heat stress tolerance could be justifiable through clear understanding of the performance of diverse mungbean genotypes for yield, yield contributing, water use efficiency and heat stress tolerance related traits.

Heterosis is the superiority of $F_{1}$ hybrid over both its parents in terms of yield or some other characters. Generally heterosis is manifested as an increased vigour, size, growth, yield or some other characteristic. The identification and enhancement of elite germplasm are essential for the ultimate success of plant breeding efforts. This phenomenon is commercially used to produce hybrid or synthetic varieties which need the maximisation of its expression and also fixation. Heterosis has been commercially utilised in cross pollinated and in some selfpollinated species.

Investigation on the magnitude of heterosis helps to identify promising hybrid combinations and also possibility of exploiting new recombinant types for yield, WUE and heat stress tolerance attributing traits from segregants. The study of heterosis will provide the basic information regarding the breeding methodology to be employed for the varietal improvement. It also helps in rejecting large number of crosses in first generation itself and selecting only those with high potential and having desired gene action for the trait of interest.

The magnitude of heterosis was reported among 21 crosses demonstrating potential of hybrid combinations of seven diverse parents for various traits enhancement in the present research. Noteworthy differences among 21 cross combinations were observed for all the 14 characters. The negative heterosis values were preferable for days to $50 \%$ flowering, days to maturity, specific leaf area and relative injury.

\section{Materials and Methods}

The present experiment was carried out at dry land farm of Sri Venkateswara Agricultural College, Tirupati, situated at an altitude of $182.9 \mathrm{~m}$. above mean sea level, $32.27^{\circ} \mathrm{N}$ latitude and $79.36^{\circ} \mathrm{E}$ longitude, situated geographically in southern agro climatic zone of Andhra Pradesh, India. The soil is sandy loam with medium fertility. Seven parents viz., ML-267, LGG-528, MGG-390, WGG-42, AKM-9904, LM-95 and EC-362096 were raised in paired row method for effecting crosses in a diallel fashion without reciprocals to generate seed of $21 \mathrm{~F}_{1}$ crosses. The $21 \mathrm{~F}_{1}$ crosses along with their seven parents constituted 28 treatments for this experiment. The seven parents and their $21 \mathrm{~F}_{1}$ crosses were sown in randomized block design with two replications during the first fortnight of November, 2016 at dry land farm, S.V. Agricultural College, Tirupati. Each genotype was sown by dibbling the seeds in two rows of $3 \mathrm{~m}$ length, with a spacing of $30 \mathrm{~cm}$ between the rows and $10 \mathrm{~cm}$ between the plants. All the 28 treatments were allotted at random to the experimental plots in each replication.The crop was fertilized at the rate of $20 \mathrm{~kg} \mathrm{~N}$ and $40 \mathrm{~kg} \mathrm{P}_{2} \mathrm{O}_{5}$ in the form of urea and single super phosphate at the time of sowing. Thinning was done to leave single seedling per hill after 15 days of sowing. Irrigation, weeding and plant protection measures were taken up as and when needed during the crop growth period, as per the standard recommended package of practices to raise a good and healthy crop. Observations were 
recorded on five randomly chosen competitive plants from each genotype in each replication for the characters viz., plant height, number of branches per plant, number of clusters per plant, pods per cluster, pods per plant, seed yield per plant, 100-seed weight, harvest index, SLA, SCMR, SLW and relative injury. Days to $50 \%$ flowering and days to maturity were recorded on plot basis. The mean values of hybrids were used for estimation of heterosis per cent under the relative heterosis, heterobeltiosis and standard heterosis categories.

\section{Results and Discussion}

The heterotic effects observed in different characters varied from cross to cross. The results of each character narrated below were presented in the Tables 1 to 5 .

In the present study, significant positive heterosis observed for seed yield per plant in most of the crosses was mainly due to the manifestation of heterosis for its component traits viz., plant height, number of branches per plant, number of clusters per plant and number of pods per plant. This would clearly indicate that heterosis for yield was through heterosis for individual yield components or additive or synergistic effects of the component characters or alternatively due to multiplicative effects of partial dominance of component characters. These results were in confirmity with the results of Aher et al., (2000a), Aher et al., (2000b), Jahagirdar et al., (2001), Loganathan et al., (2001), Dethe and Patil (2008), Patel et al., (2009), Sathya and Jayamani (2011), Bhagora et al., (2013) and Srivastava and Singh (2013). From the above discussion of the results obtained in the present study, it is interesting to note that the various levels of positive and negative heterosis was observed for various characters in $F_{1}$ over the parental mean. The level of heterosis exhibited in the $F_{1} \mathrm{~S}$ is attributed to the presence of over dominance and increased parental diversity in the expression of heterosis. In high heterotic crosses increase in number of clusters per plant and number of pods per plant associated with increase in seed yield. The present study indicated low heterosis in some of the cross combinations which can be improved by using genetically variable germplasm of mungbean. Consequently, the crosses exhibiting significant high heterosis should be exploited to develop high yielding varieties of mungbean. The promising crosses have enormous potential to make use of the heterosis or to isolate desirable segregants.

Based on heterobeltiosis studies, the crosses viz., MGG $390 \times$ LM 95 for plant height and number of branches per plant; WGG $42 \times$ LM 95 for days to $50 \%$ flowering and days to maturity; ML $267 \times$ LGG 528 for number of clusters per plant, number of pods per plant, seed yield per plant and harvest index; ML $267 \times$ WGG 42 for number of pods per cluster; WGG $42 \times$ AKM 9904 for 100 seed weight were found to be good cross combinations for the respective yield attributing traits.

The cross LM $95 \times$ EC 362096 for specific leaf area and specific leaf weight; WGG $42 \times$ EC 362096 for SCMR were found to be good cross combinations for respective WUE related traits. For heat stress tolerance related trait like relative injury the cross LM $95 \times \mathrm{EC}$ 362096 was rated as good cross combination. 
Table.1 Percentage of Relative heterosis (RH), Heterobeltiosis (HB) and Standard heterosis (SH) for days to 50\% flowering, days to maturity and plant height in mungbean

\begin{tabular}{|c|c|c|c|c|c|c|c|c|c|c|}
\hline \multirow[t]{2}{*}{ S. No. } & \multirow[t]{2}{*}{ Crosses } & \multicolumn{3}{|c|}{ Days to $50 \%$ flowering } & \multicolumn{3}{|c|}{ Days to maturity } & \multicolumn{3}{|c|}{ Plant height (cm) } \\
\hline & & RH & HB & SH & RH & HB & SH & RH & HB & SH \\
\hline 1 & ML $267 \times$ LGG 528 & -4.17 & -2.82 & -5.48 & $-3.32 *$ & -1.50 & $-5.07 * *$ & $8.08 * *$ & 3.35 & $13.27 * *$ \\
\hline 2 & ML $267 \times$ MGG 390 & $15.07 * *$ & $18.31 * *$ & $15.07 * *$ & 2.21 & $4.51 * *$ & 0.72 & 4.28 & 2.05 & $11.84 * *$ \\
\hline 4 & ML $267 \times$ AKM 9904 & 1.32 & $8.45^{*}$ & 5.48 & -0.72 & 3.01 & -0.72 & 0.57 & -1.12 & $8.37 * *$ \\
\hline 5 & ML $267 \times$ LM 95 & -1.30 & $7.04 *$ & 4.11 & 0.72 & $5.26 * *$ & 1.45 & 3.63 & 0.93 & $10.61 * *$ \\
\hline 6 & ML $267 \times$ EC 362096 & $10.67 * *$ & $16.90 * *$ & $13.70 * *$ & 0.74 & 2.26 & -1.45 & 2.21 & 0.91 & $13.47 * *$ \\
\hline 8 & LGG $528 \times$ WGG 42 & -0.65 & 5.48 & 5.48 & $3.94 * *$ & $5.07 * *$ & $5.07 * *$ & 2.36 & -2.45 & -2.45 \\
\hline 9 & LGG $528 \times$ AKM 9904 & $7.79 * *$ & $13.70 * *$ & $13.70 * *$ & -1.78 & 0.00 & 0.00 & 4.06 & 1.16 & $7.14 *$ \\
\hline 10 & LGG $528 \times$ LM 95 & -1.28 & 5.48 & 5.48 & $-3.89 * *$ & -1.45 & -1.45 & 3.30 & 1.38 & 5.31 \\
\hline 11 & LGG $528 \times$ EC 362096 & 1.32 & 5.48 & 5.48 & -0.36 & 0.00 & -0.72 & 3.94 & -1.81 & $10.41 * *$ \\
\hline 12 & MGG $390 \times$ WGG 42 & -3.18 & 1.33 & 4.11 & $-5.71 * *$ & $-5.04 * *$ & $-4.35 * *$ & $6.26^{*}$ & -0.97 & 3.88 \\
\hline 13 & MGG $390 \times$ AKM 9904 & -3.85 & 0.00 & 2.74 & $-4.26 * *$ & -2.88 & -2.17 & 1.84 & 1.35 & $7.35^{*}$ \\
\hline 17 & WGG $42 \times$ LM 95 & $-18.79 * *$ & $-18.29 * *$ & $-8.22 *$ & $-7.69 * *$ & $-6.38 * *$ & $-4.35 * *$ & 4.72 & -1.96 & 1.84 \\
\hline 18 & WGG $42 \times$ EC 362096 & $-14.29 * *$ & $-12.66 * *$ & -5.48 & -2.16 & -0.73 & -1.45 & 3.52 & $-6.53 *$ & 5.10 \\
\hline 19 & AKM $9904 \times$ LM 95 & $-8.54 * *$ & $-7.41 *$ & 2.74 & -0.69 & 0.00 & $3.62 *$ & 4.47 & 3.47 & $9.58 * *$ \\
\hline 20 & AKM 9904 × EC 362096 & $-10.00 * *$ & $-8.86 * *$ & -1.37 & 0.71 & 2.92 & 2.17 & -1.50 & -4.36 & $7.55^{*}$ \\
\hline 21 & LM $95 \times$ EC 362096 & $-8.64 * *$ & $-6.33 *$ & 1.37 & -1.42 & 1.46 & 0.72 & $7.55^{* *}$ & 3.45 & $16.33 * *$ \\
\hline & S.E & 0.96 & 1.11 & 1.11 & 0.86 & 0.99 & 0.99 & 1.16 & 1.34 & 1.34 \\
\hline
\end{tabular}

* Significant at $5 \%$ level, ** Significant at $1 \%$ level 
Table.2 Percentage of Relative heterosis (RH), Heterobeltiosis (HB) and Standard heterosis (SH) for number of branches per plant, number of clusters per plant and number of pods per cluster in mungbean

\begin{tabular}{|c|c|c|c|c|c|c|c|c|c|c|}
\hline \multirow{2}{*}{$\begin{array}{l}\text { S. } \\
\text { No. }\end{array}$} & \multirow[t]{2}{*}{ Crosses } & \multicolumn{3}{|c|}{ Number of branches per plant } & \multicolumn{3}{|c|}{ Number of clusters per plant } & \multicolumn{3}{|c|}{ Number of pods per cluster } \\
\hline & & RH & HB & SH & RH & HB & SH & RH & HB & SH \\
\hline 1 & ML $267 \times$ LGG 528 & $52.00 * *$ & 14.00 & $128.00 * *$ & $57.09 * *$ & $50.39 * *$ & $64.41 * *$ & $26.70 *$ & 10.82 & 10.82 \\
\hline 2 & ML $267 \times$ MGG 390 & -24.05 & $-40.00 * *$ & 20.00 & $21.61 * *$ & $15.28 *$ & $40.68 * *$ & 19.78 & 6.22 & 2.88 \\
\hline 3 & ML $267 \times$ WGG 42 & 5.56 & -24.00 & 52.00 & $17.74 *$ & 14.71 & $32.20 * *$ & $42.31 * *$ & $40.79 * *$ & 7.81 \\
\hline 4 & ML $267 \times$ AKM 9904 & $-38.03 *$ & $-56.00 * *$ & -12.00 & $47.62 * *$ & $44.19 * *$ & $57.63 * *$ & 15.40 & 0.41 & 1.64 \\
\hline 5 & ML $267 \times$ LM 95 & -11.69 & $-32.00 *$ & 36.00 & $39.92 * *$ & $37.31 * *$ & $55.93 * *$ & $46.52 * *$ & $32.54 *$ & 22.74 \\
\hline 6 & ML $267 \times$ EC 362096 & -6.85 & $-32.00 *$ & 36.00 & $29.08 * *$ & $25.58 * *$ & $37.29 * *$ & $32.93 *$ & 25.20 & 6.16 \\
\hline 7 & LGG $528 \times$ MGG 390 & -14.81 & -20.69 & -8.00 & $29.77 * *$ & $18.06^{*}$ & $44.07 * *$ & -2.44 & -3.97 & -3.97 \\
\hline 8 & LGG $528 \times$ WGG 42 & 10.64 & 4.00 & 4.00 & $22.83 * *$ & 14.71 & $32.20 * *$ & 17.15 & 3.42 & 3.42 \\
\hline 9 & LGG $528 \times$ AKM 9904 & 13.04 & 4.00 & 4.00 & $41.08 * *$ & $38.21 * *$ & $44.07 * *$ & $-26.62 *$ & $-27.06^{*}$ & $-26.16^{*}$ \\
\hline 10 & LGG $528 \times$ LM 95 & 3.85 & 0.00 & 8.00 & $40.48 * *$ & $32.09 * *$ & $50.00 * *$ & 0.71 & -3.01 & -3.01 \\
\hline 11 & LGG $528 \times$ EC 362096 & 16.67 & 12.00 & 12.00 & $51.67 * *$ & $49.18 * *$ & $54.24 * *$ & 0.52 & -7.12 & -7.12 \\
\hline 12 & MGG $390 \times$ WGG 42 & $52.94 *$ & $34.48 *$ & $56.00 *$ & $19.29 * *$ & $15.97 *$ & $41.53 * *$ & -0.95 & -11.32 & -14.11 \\
\hline 13 & MGG $390 \times$ AKM 9904 & -12.00 & -24.14 & -12.00 & $21.35 * *$ & 12.50 & $37.29 * *$ & -6.36 & -8.39 & -7.26 \\
\hline 14 & MGG $390 \times$ LM 95 & $64.29 * *$ & $58.62 *$ & $84.00 * *$ & $35.25 * *$ & $30.56 * *$ & $59.32 * *$ & $25.09 *$ & 22.35 & 18.49 \\
\hline 15 & MGG $390 \times$ EC 362096 & -3.85 & -13.79 & 0.00 & $24.81 * *$ & $15.28^{*}$ & $40.68 * *$ & $25.79 *$ & 17.96 & 14.25 \\
\hline 16 & WGG $42 \times$ AKM 9904 & -2.33 & -4.55 & -16.00 & 1.93 & -2.94 & 11.86 & -12.94 & -23.55 & -22.60 \\
\hline 17 & WGG $42 \times$ LM 95 & -14.29 & -22.22 & -16.00 & -2.96 & -3.68 & 11.02 & -12.71 & -20.27 & $-26.16^{*}$ \\
\hline 18 & WGG $42 \times$ EC 362096 & 6.67 & 4.35 & -4.00 & $-20.93 * *$ & $-25.00 * *$ & -13.56 & 6.62 & 1.45 & -13.97 \\
\hline 19 & AKM $9904 \times$ LM 95 & $58.33^{*}$ & 40.74 & $52.00^{*}$ & -1.17 & -5.22 & 7.63 & -8.13 & -12.04 & -10.96 \\
\hline 20 & $\begin{array}{c}\text { AKM } 9904 \times \mathrm{EC} \\
362096\end{array}$ & 27.27 & 21.74 & 12.00 & 13.47 & 13.01 & $17.80 *$ & 13.70 & 4.47 & 5.75 \\
\hline 21 & LM $95 \times$ EC 362096 & $60.00 *$ & 48.15 & $60.00^{*}$ & $47.66 * *$ & $41.04 * *$ & $60.17 * *$ & 4.40 & 0.00 & -7.40 \\
\hline & S.E. & 0.27 & 0.32 & 0.32 & 0.43 & 0.50 & 0.50 & 0.38 & 0.44 & 0.44 \\
\hline
\end{tabular}

* Significant at 5\% level, ** Significant at $1 \%$ level 
Table.3 Percentage of Relative heterosis (RH), Heterobeltiosis (HB) and Standard heterosis (SH) for number of pods per plant, hundred seed weight and harvest index in mungbean

\begin{tabular}{|c|c|c|c|c|c|c|c|c|c|c|}
\hline \multirow{2}{*}{$\begin{array}{c}\text { S. } \\
\text { No. }\end{array}$} & \multirow[t]{2}{*}{ Crosses } & \multicolumn{3}{|c|}{ Number of pods per plant } & \multicolumn{3}{|c|}{ 100-seed weight (g) } & \multicolumn{3}{|c|}{ Harvest index (\%) } \\
\hline & & RH & HB & SH & RH & HB & SH & RH & HB & SH \\
\hline 1 & ML $267 \times$ LGG 528 & $100.00 * *$ & $82.33 * *$ & $82.33 * *$ & 7.24 & 5.61 & 8.93 & $31.31 * *$ & $31.04 * *$ & $31.58 * *$ \\
\hline 2 & ML $267 \times$ MGG 390 & $44.32 * *$ & $22.44 *$ & $44.65 * *$ & $23.13 * *$ & $20.73 *$ & $24.53 * *$ & 6.14 & 2.13 & 10.94 \\
\hline 3 & ML $267 \times$ WGG 42 & $66.12 * *$ & $60.85 * *$ & $41.40 * *$ & -7.07 & -8.71 & -2.39 & $19.65 *$ & 16.50 & $16.98 *$ \\
\hline 4 & ML $267 \times$ AKM 9904 & $71.22 * *$ & $52.65 * *$ & $60.47 * *$ & 3.68 & 3.17 & 6.42 & -2.32 & -9.16 & 6.06 \\
\hline 5 & ML $267 \times$ LM 95 & $104.21 * *$ & $81.90 * *$ & $91.63 * *$ & 13.11 & 3.66 & 6.92 & 11.41 & 11.37 & 11.91 \\
\hline 6 & ML $267 \times$ EC 362096 & $72.53 * *$ & $67.91 * *$ & $46.05 * *$ & -5.26 & $-13.27 *$ & 7.67 & 8.77 & 7.34 & 7.79 \\
\hline 7 & LGG $528 \times$ MGG 390 & $27.08 * *$ & 17.32 & $38.60 * *$ & 9.41 & 8.93 & 8.93 & -0.63 & -4.58 & 3.65 \\
\hline 8 & LGG $528 \times$ WGG 42 & $46.04 * *$ & $37.21 * *$ & $37.21 * *$ & -4.80 & -7.88 & -1.51 & 14.14 & 11.35 & 11.35 \\
\hline 9 & LGG $528 \times$ AKM 9904 & 4.31 & 1.77 & 6.98 & 7.90 & 6.77 & 9.06 & -0.04 & -7.21 & 8.34 \\
\hline 10 & LGG $528 \times$ LM 95 & $41.79 * *$ & $38.19 * *$ & $45.58 * *$ & -4.60 & -11.32 & -11.32 & 8.67 & 8.41 & 8.93 \\
\hline 11 & LGG $528 \times$ EC 362096 & $52.74 * *$ & $42.79 * *$ & $42.79 * *$ & 5.39 & -4.86 & $18.11^{*}$ & 3.16 & 2.01 & 2.01 \\
\hline 12 & MGG $390 \times$ WGG 42 & 17.70 & 2.64 & 21.26 & 0.85 & -2.82 & 3.90 & -2.60 & -8.65 & -0.77 \\
\hline 13 & MGG $390 \times$ AKM 9904 & 14.17 & 7.87 & $27.44 *$ & -0.13 & -1.60 & 0.50 & -4.19 & -7.53 & 7.96 \\
\hline 14 & MGG $390 \times$ LM 95 & $68.99 * *$ & $58.84 * *$ & $88.84 * *$ & $22.77 * *$ & $14.59 *$ & 13.58 & $21.23 * *$ & $16.68 *$ & $26.75^{* *}$ \\
\hline 15 & MGG $390 \times$ EC 362096 & $56.92 * *$ & $36.22 * *$ & $60.93 * *$ & $-14.37 *$ & $-23.00 * *$ & -4.40 & 5.78 & 0.49 & 9.16 \\
\hline 16 & WGG $42 \times$ AKM 9904 & -11.33 & -18.58 & -14.42 & $38.27 * *$ & $35.18 * *$ & $44.53 * *$ & 4.72 & -4.98 & 10.94 \\
\hline 17 & WGG $42 \times$ LM 95 & -15.28 & -22.30 & -18.14 & $39.47 * *$ & $25.76 * *$ & $34.47 * *$ & 11.18 & 8.21 & 8.74 \\
\hline 18 & WGG $42 \times$ EC 362096 & -14.89 & -15.34 & $-25.58 *$ & $32.28 * *$ & $23.10 * *$ & $52.83 * *$ & 2.61 & 1.23 & -1.03 \\
\hline 19 & AKM $9904 \times$ LM 95 & -9.83 & -9.93 & -5.12 & 2.88 & -5.30 & -3.27 & -8.58 & $-14.95 *$ & -0.70 \\
\hline 20 & $\begin{array}{c}\text { AKM } 9904 \times \text { EC } \\
362096\end{array}$ & $27.85^{*}$ & 16.81 & 22.79 & 6.95 & -2.53 & $21.01 *$ & 2.83 & -5.54 & 10.29 \\
\hline 21 & LM $95 \times$ EC 362096 & $54.29 * *$ & $40.84 * *$ & $48.37 * *$ & $30.78 * *$ & 10.64 & $37.36 * *$ & $32.05 * *$ & $30.27 * *$ & $30.89 * *$ \\
\hline & S.E. & 2.16 & 2.5 & 2.5 & 0.27 & 0.31 & 0.31 & 2.56 & 2.96 & 2.96 \\
\hline
\end{tabular}

* Significant at 5\% level, ** Significant at $1 \%$ level 
Table.4 Percentage of Relative heterosis (RH), Heterobeltiosis (HB) and Standard heterosis (SH) for SPAD chlorophyll meter reading (SCMR), specific leaf area and specific leaf weight in mungbean

\begin{tabular}{|c|c|c|c|c|c|c|c|c|c|c|}
\hline \multirow{2}{*}{$\begin{array}{c}\text { S.No } \\
\text {. }\end{array}$} & \multirow[t]{2}{*}{ Crosses } & \multicolumn{3}{|c|}{ SCMR } & \multicolumn{3}{|c|}{ Specific leaf area $\left(\mathrm{cm}^{2} \mathrm{~g}^{-1}\right)$} & \multicolumn{3}{|c|}{ Specific leaf weight $\left(\mathrm{g} \mathrm{cm}^{-2}\right)$} \\
\hline & & RH & HB & SH & RH & HB & SH & RH & HB & SH \\
\hline 1 & ML $267 \times$ LGG 528 & 6.77 & 6.36 & 6.36 & $11.48 * *$ & -3.30 & -3.30 & $12.11 * *$ & 3.31 & 3.31 \\
\hline 2 & ML $267 \times$ MGG 390 & 6.87 & 4.89 & 4.10 & 1.15 & 4.01 & $16.64 * *$ & -0.95 & -3.70 & $-14.05 * *$ \\
\hline 3 & ML $267 \times$ WGG 42 & -1.98 & -3.26 & -3.99 & $9.30 * *$ & $14.11 * *$ & $24.26 * *$ & $-8.92 * *$ & $-12.61 * *$ & $-19.83 * *$ \\
\hline 4 & ML $267 \times$ AKM 9904 & -0.16 & -0.33 & -1.08 & $5.43 *$ & $7.34^{*}$ & $22.72 * *$ & -4.81 & $-6.60 *$ & $-18.18 * *$ \\
\hline 5 & ML $267 \times$ LM 95 & -2.03 & -2.93 & -3.67 & 3.88 & 4.72 & $24.07 * *$ & -4.43 & -4.90 & $-19.83 * *$ \\
\hline 6 & ML $267 \times$ EC 362096 & 4.68 & 3.26 & 2.48 & -0.46 & $14.55 * *$ & 4.26 & -0.85 & $-12.12 * *$ & -4.13 \\
\hline 7 & LGG $528 \times$ MGG 390 & -5.79 & -7.87 & -7.87 & 4.13 & $10.45 * *$ & $10.45 * *$ & -4.80 & $-9.92 * *$ & $-9.92 * *$ \\
\hline 8 & LGG $528 \times$ WGG 42 & -1.48 & -3.13 & -3.13 & $7.28 * *$ & $12.06 * *$ & $12.06 * *$ & $-6.90 * *$ & $-10.74 * *$ & $-10.74 * *$ \\
\hline 9 & LGG $528 \times$ AKM 9904 & -6.40 & -6.90 & -6.90 & $13.14 * *$ & $21.24 * *$ & $21.24 * *$ & $-11.89 * *$ & $-17.36 * *$ & $-17.36 * *$ \\
\hline 10 & LGG $528 \times$ LM 95 & 5.68 & 4.31 & 4.31 & $14.43 * *$ & $26.10 * *$ & $26.10 * *$ & $-13.51 * *$ & $-20.66^{* *}$ & $-20.66 * *$ \\
\hline 11 & LGG $528 \times$ EC 362096 & 4.61 & 2.80 & 2.80 & $12.91 * *$ & $18.48 * *$ & $7.84^{*}$ & $-11.46^{* *}$ & $-15.15 * *$ & $-7.44 * *$ \\
\hline 12 & MGG $390 \times$ WGG 42 & 0.11 & -0.45 & -3.78 & -0.86 & 0.62 & $9.57 * *$ & 1.37 & 0.00 & $-8.26 * *$ \\
\hline 13 & MGG $390 \times$ AKM 9904 & 1.05 & -0.65 & -1.73 & 2.19 & 3.18 & $15.71 * *$ & -2.80 & -3.70 & $-14.05^{* *}$ \\
\hline 14 & MGG $390 \times$ LM 95 & $9.56^{*}$ & 8.53 & 5.72 & $-6.42 * *$ & -2.98 & $8.79 * *$ & $6.22 *$ & 2.78 & $-8.26 * *$ \\
\hline 15 & MGG $390 \times$ EC 362096 & -3.87 & -4.36 & -7.66 & $-8.31 * *$ & 2.33 & $-6.86^{*}$ & $8.33 * *$ & -1.52 & $7.44 * *$ \\
\hline 16 & WGG $42 \times$ AKM 9904 & $11.86 * *$ & $10.58 *$ & $9.39 *$ & 1.04 & 3.56 & $12.77 * *$ & -1.38 & -3.60 & $-11.57 * *$ \\
\hline 17 & WGG $42 \times$ LM 95 & 5.95 & 5.54 & 2.80 & 3.53 & $8.99 * *$ & $18.69 * *$ & -3.77 & $-8.11 * *$ & $-15.70 * *$ \\
\hline 18 & WGG $42 \times$ EC 362096 & $18.03 * *$ & $17.97 * *$ & $14.02 * *$ & $16.00 * *$ & $27.40 * *$ & $15.95 * *$ & $-14.40 * *$ & $-21.21 * *$ & $-14.05 * *$ \\
\hline 19 & AKM $9904 \times$ LM 95 & -7.25 & -7.96 & -8.95 & -0.10 & 2.55 & $17.25 * *$ & -0.48 & -2.83 & $-14.88 * *$ \\
\hline 20 & $\begin{array}{c}\text { AKM } 9904 \times \mathrm{EC} \\
362096\end{array}$ & 3.97 & 2.73 & 1.62 & $10.98 * *$ & $25.20 * *$ & $13.95 * *$ & $-10.92 * *$ & $-19.70 * *$ & $-12.40 * *$ \\
\hline 21 & LM $95 \times$ EC 362096 & 6.79 & 6.31 & 3.56 & $-16.80 * *$ & -3.37 & $\frac{-}{-}$ & $18.45 * *$ & 4.55 & $14.05 * *$ \\
\hline & S.E. & 1.63 & 1.89 & 1.89 & 4.35 & 5.03 & 5.03 & 0.00014 & 0.00016 & 0.00016 \\
\hline
\end{tabular}

* Significant at $5 \%$ level, ** Significant at $1 \%$ level 
Table.5 Percentage of Relative heterosis (RH), Heterobeltiosis (HB) and Standard heterosis (SH) for relative injury and seed yield per plant in mungbean

\begin{tabular}{|c|c|c|c|c|c|c|c|}
\hline \multirow[t]{2}{*}{ S. No. } & \multirow[t]{2}{*}{ Crosses } & \multicolumn{3}{|c|}{ Relative injury (\%) } & \multicolumn{3}{|c|}{ Seed yield per plant (g) } \\
\hline & & RH & HB & SH & RH & HB & SH \\
\hline 1 & ML $267 \times$ LGG 528 & $-26.85 * *$ & $-23.99 * *$ & $-29.51 * *$ & $67.11 * *$ & $61.85 * *$ & $61.85 * *$ \\
\hline 2 & ML $267 \times$ MGG 390 & 0.92 & 3.84 & -3.70 & 4.33 & -8.27 & 13.34 \\
\hline 3 & ML $267 \times$ WGG 42 & 5.93 & $9.97 *$ & 1.98 & 14.99 & 13.65 & 9.04 \\
\hline 4 & ML $267 \times$ AKM 9904 & $-8.84 *$ & 2.50 & -4.95 & $38.94 * *$ & $31.47 * *$ & $38.03 * *$ \\
\hline 5 & ML $267 \times$ LM 95 & $16.77 * *$ & $24.70 * *$ & $15.64 * *$ & $54.19 * *$ & $49.07 * *$ & $49.63 * *$ \\
\hline 6 & ML $267 \times$ EC 362096 & $-7.28 *$ & -2.13 & $-9.24 *$ & 17.02 & 9.37 & 17.89 \\
\hline 7 & LGG $528 \times$ MGG 390 & $13.19 * *$ & $14.29 * *$ & $12.12 * *$ & 1.12 & -8.53 & 13.03 \\
\hline 8 & LGG $528 \times$ WGG 42 & $18.12 * *$ & $18.24 * *$ & $17.99 * *$ & 16.89 & 14.53 & 14.53 \\
\hline 9 & LGG $528 \times$ AKM 9904 & 4.10 & $12.33 * *$ & $12.33 * *$ & 1.82 & -0.59 & 4.36 \\
\hline 10 & LGG $528 \times$ LM 95 & $7.56^{*}$ & $10.43^{*}$ & $10.43 *$ & $19.60 *$ & 19.38 & 19.83 \\
\hline 11 & LGG $528 \times$ EC 362096 & $-20.84 * *$ & -19.64 & $-19.64 * *$ & 8.85 & 4.92 & 13.09 \\
\hline 12 & MGG $390 \times$ WGG 42 & -5.32 & -4.51 & -6.32 & -3.44 & -14.23 & 5.99 \\
\hline 13 & MGG $390 \times$ AKM 9904 & $-6.67 *$ & 1.76 & -0.17 & -3.16 & -10.44 & 10.66 \\
\hline 14 & MGG $390 \times$ LM 95 & $-27.89 * *$ & $-25.24 * *$ & $-26.65 * *$ & $48.55 * *$ & $34.61 * *$ & $66.33 * *$ \\
\hline 15 & MGG $390 \times$ EC 362096 & $14.74 * *$ & $17.62 * *$ & $15.39 * *$ & $15.76^{*}$ & 8.38 & $33.92 * *$ \\
\hline 16 & WGG $42 \times$ AKM 9904 & $7.22 *$ & $15.82 * *$ & $15.58 * *$ & 3.32 & -1.13 & 3.80 \\
\hline 17 & WGG $42 \times$ LM 95 & $12.78 * *$ & $15.91 * *$ & $15.67 * *$ & 4.29 & 1.99 & 2.37 \\
\hline 18 & WGG $42 \times$ EC 362096 & $8.87 *$ & $10.64^{*}$ & $10.41 *$ & $-18.42 *$ & $-22.90 *$ & -16.90 \\
\hline 19 & AKM $9904 \times$ LM 95 & $8.74 *$ & $14.14 * *$ & $20.23 * *$ & -13.36 & -15.26 & -11.03 \\
\hline 20 & AKM $9904 \times$ EC 362096 & $-34.53 * *$ & $-30.47 * *$ & $-28.37 * *$ & 9.99 & 8.56 & 17.02 \\
\hline 21 & LM $95 \times$ EC 362096 & $-37.89 * *$ & $-37.19 * *$ & $-35.29 * *$ & $57.89 * *$ & $52.46 * *$ & $64.34 * *$ \\
\hline & S.E & 1.46 & 1.68 & 1.68 & 0.69 & 0.80 & 0.80 \\
\hline
\end{tabular}

* Significant at $5 \%$ level ** Significant at $1 \%$ level 
Studies on standard heterosis revealed that the cross LM $95 \times$ EC 362096 was adjudged as the best as it expressed significant heterosis in desirable direction for seven yield traits $v i z$, plant height, number of branches per plant, number of clusters per plant, number of pods per plant, 100 seed weight, harvest index and seed yield per plant along with two WUE related traits like SLA and SLW and heat stress tolerance related trait relative injury. Similarly, the cross MGG $390 \times$ LM 95 was considered as second best as it expressed significant standard heterosis in desirable direction for six yield traits viz., plant height, number of branches per plant, number of clusters per plant, number of pods per plant, harvest index and seed yield per plant along with two WUE related traits like SLA and SLW and heat stress tolerance related trait relative injury.

The cross ML $267 \times$ LGG 528 has shown significant heterosis in desirable direction for plant height, number of branches per plant, number of clusters per plant, number of pods per plant, harvest index and seed yield per plant along with heat stress tolerance related trait relative injury. Hence, these crosses could be exploited for obtaining transgressive segregants in advanced generations with increased seed yield per plant and drought and heat tolerance ability.

The present study also confirmed that high heterotic combinations were realized in the cross combinations involving the genetically diverse parents for seed yield and its components. Further, all the heterotic cross combinations had close correspondence with mean value, which suggested that the per se performance of crosses could be considered for judging heterosis for seed yield per plant.

From the above discussion, it is concluded that the crosses viz., LM $95 \times$ EC 362096, MGG $390 \times$ LM 95 and ML $267 \times$ LGG 528 were identified as best for majority of the yield, water use efficiency and heat stress tolerant traits. Hence, these crosses could be suggested for exploiting in future breeding programmes.

\section{References}

Aher, R.P., Sonawane, V.P and Dahat, D.V. 2000a. Heterosis in mungbean (Vigna radiata (L.) Wilczek). Indian Journal of Agricultural Research. 34(2): 134137.

Aher, R.P., Surve, P.P and Dahat, D.V. 2000b. Heterosis for yield and yield components in mungbean (Vigna radiata (L.) Wilczek). Agricultural Science Digest. 20(1): 5-8.

Bhagora, N.B., Nizama, J.R and Patel, S.R. 2013. Hybrid vigour study in mungbean [Vigna radiata (L.) Wilczek]. Asian Resonance. 2(4): 5155.

Dethe, A.M and Patil, J.V. 2008. Heterosis studies in mungbean (Vigna radiata (L.) Wilczek). Legume research. 31(1): 36-39.

Jahagirdar, J.E. 2001. Heterosis and combining ability studies for seed yield and yield components in mungbean (Vigna radiata (L.) Wilczek). Indian Journal of Pulses Research. 14(2): 141-142.

Loganathan, P., Saravanan, K., Thangavel, P and Ganesan, J. 2001. Heterosis for yield and yield components in green gram (Vigna radiata (L) Wilczek). Legume Research. 24(2): 77-81.

Patel, M.B., Patel, B.N., Salviya, J.J and Tikka, S.B.S. 2009. Heterosis and genetic architecture of yield, yield contributing traits and yellow mosaic virus in mungbean (Vigna radiata $(\mathrm{L})$ Wilczek). Legume research. 32(4): 260-264.

Sathya, M and Jayamani, P. 2011. Heterosis and combining ability studies in green 
gram. Journal of Food Legumes. 24(4): 282-287.Srivastava, R.L and Singh, G. 2013. Heterosis for yield and its contributing characters in mungbean (Vigna radiate (L.) wilczek). Indian Journal of Science Research. 4(1): 131-134.

\section{How to cite this article:}

Reddy, B.R.K., K.H.P. Reddy, D.M. Reddy, P. Sudhakar and Ravindra Reddy, B. 2019. Heterosis for Yield, Yield Attributes and Water use Efficiency related Traits in Mungbean. Int.J.Curr.Microbiol.App.Sci. 8(12): 634-643. doi: https://doi.org/10.20546/ijcmas.2019.812.083 